REVISTA INTERNACIONAL DE CIENCIAS DEL DEPORTE International Journal of Sport Science

Rev. int. cienc. deporte

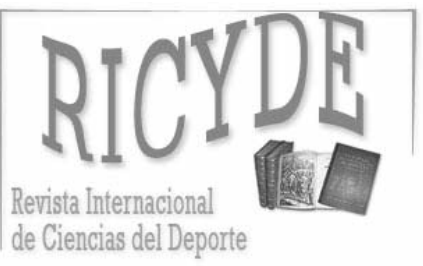

International Journal of Sport Science Volumen VI - Año VI

Páginas:154-165 ISSN:1885-3137

No 19 - Abril - 2010

\title{
Relación entre eficacia, lateralidad y zona de lanzamiento del penalti en función del nivel de competición en fútbol. \\ Relationship between efficacy, laterality of foot strike, and shot zone of the penalty in relation to competition level in soccer.
}

\author{
José Manuel Palao \\ Manuel López-Montero \\ Facultad de Ciencias de la Salud, Actividad Física y Deporte. \\ Universidad Católica de San Antonio (Murcia, España) \\ Manuel López-Botella \\ Instituto Enseñanza Secundaria "La Mola" (Novelda. España)
}

\section{Resumen}

El objetivo del presente trabajo fue conocer cómo varía la lateralidad, la zona de lanzamiento y la eficacia del penalti en lanzadores especialistas profesionales de fútbol en función del nivel de la competición. La muestra estudiada fue de 642 penaltis de competiciones profesionales españolas de 1 a División, $2^{a}$ División A, y $2^{a}$ División $B$ de las temporadas 2004/2005, 2005/2006 y 2006/2007. Se realizó un diseño de investigación descriptivo correlacional intra e intergrupo. Las variables objeto de estudio fueron: resultado del lanzamiento (gol, parado, poste, y fuera), lateralidad del lanzador, zona de destino, y nivel de competición (1 $1^{a}$ División, $2^{a}$ División $A$, y $2^{a}$ División $B$ ). Se realizó un análisis de los datos descriptivo e inferencial (Test de Chi-cuadrado y tablas de contingencia). A partir de los resultados obtenidos se pueden extraer las siguientes conclusiones: los lanzadores de penalti analizados obtienen gol en siete de cada 10 lanzamientos. Los diestros tienden a lanzar a la derecha del portero, y los zurdos a la izquierda del portero. Tanto diestros como zurdos son más efectivos lanzando a la derecha del portero. Los lanzamientos dirigidos a las zonas altas presentan un $10 \%$ mayor de eficacia que los lanzamientos dirigidos a las zonas bajas. Ninguno de los lanzamientos dirigidos a las zonas altas de la portería fue parado por el portero. A menor nivel de la competición, menos lanzamientos a las zonas altas.

Palabras clave: análisis de juego; fútbol; penaltis; nivel de rendimiento.

\begin{abstract}
The purpose of this paper was to study the relationship between competition level, laterality of foot strike, and shot zone on penalty efficacy in professional penalty-taking specialist football players. A total of 642 penalty shots from the Spanish professional divisions (first, second, and third levels of competition) in the 2004-2005, 2005-2006 and 2006-2007 seasons were analyzed. A descriptive and correlational intra- and inter-group study design was used. The variables studied were: shot efficacy (goals, saves by the goalkeeper, hitting the goal post, and misses), foot used for strike, shot zone, and competition level. Descriptive and inferential analyses (Chi-Square Test and likelihood ratio) of the data were done. The following conclusions can be established from the results obtained: penalty takers obtain goals in seven out of ten shots; right-footed players tend to shoot more to the right of the goalkeeper and left-footed players shoot more to the left of the goalkeeper; both right-footed and left-footed players were more effective shooting to the right of the goalkeeper; higher shots had $10 \%$ more efficacy than lower shots; none of the shots to the high part of the goal were stopped by the goalkeeper; and at lower levels of competitions, there were fewer shots to the high part of the goal.
\end{abstract}

Key words: match analysis; football; penalties; performance. 
Palau, J.M.; López-Montero, M.; López-Botella, M. (2010). Relación entre eficacia, lateralidad, y zona de lanzamiento del penalti en función del nivel de competición en fútbol. Revista Internacional de Ciencias del Deporte. 19(6), 153-66. http://www.cafyd.com/REVISTA/01905.pdf

\section{Introducción}

$\mathrm{E}$ n el deporte de rendimiento, la competición es la referencia para el establecimiento de las formas, medios y criterios de entrenamiento. Los deportes colectivos presentan más dificultades en el establecimiento de dichos criterios que los deportes individuales al haber más factores que afectan al rendimiento. Para planificar el entrenamiento de forma adecuada es necesario conocer cómo realizan los jugadores su actuación en competición. En fútbol, las distintas acciones del juego implican diferentes jugadores e interacciones entre estos. El penalti es una de las pocas acciones de juego en la cual se ven implicados únicamente dos jugadores: el lanzador y el portero. Si se atiende a la forma de realizar el penalti por parte del lanzador se pueden diferenciar dos grandes opciones:

a) El lanzador ejecuta su acción sin tener en cuenta la actuación del portero. El jugador lanza a su/s zona/s habituales y/o a las que obtiene mayor eficacia.

b) El lanzador ejecuta su acción teniendo en cuenta la actuación del portero. El jugador busca enviar el balón a la zona contraria a donde se ha tirado o va a tirarse el portero.

El objetivo final en ambas es el mismo, conseguir gol, pero éste se busca de diferente forma. En la primera opción se busca precisión y/o potencia en el lanzamiento, mientras que en la segunda opción se busca engañar o esconder el gesto. El presente trabajo de investigación se centra en profundizar en las conductas que realiza el lanzador, no estudiándose si el lanzador tiene en cuenta o no la actuación del portero. El conocimiento de cuáles son las formas de ejecución más eficaces se hace necesario para un trabajo adecuado de esta acción en los entrenamientos.

Los estudios realizados sobre esta temática muestran indirectamente la existencia de diferencias entre lanzadores especialistas y lanzadores de tandas de penaltis en la eficacia de lanzamiento (Castillo, Oña, Raya, y Martínez, 2002; Hughes y Wells, 2001; Miller, 1996; Morya, Bigatao, Lees, y Ranvaud, 2003; López-Botella y Palao, 2007; Vázquez, 1998). Los especialistas presentan una mayor eficacia (8 de cada 10 lanzamientos son gol) que los lanzadores de tandas (7 de cada 10 lanzamientos son gol). Teniendo en cuenta la lateralidad, tanto diestros como zurdos presentan valores de eficacia similares. Los estudios revisados presentan ligeras diferencias del $2 \%$ en función de la lateralidad (Hughes y Wells, 2001; López-Botella y Palao, 2007). No obstante, sí se ha descrito que los lanzadores diestros presentan una tendencia a lanzar a la zona derecha de la portería (López-Botella y Palao, 2007; Raya y Navarro, 1990) y que los lanzadores zurdos presentan una tendencia a lanzar a la zona izquierda de la portería (López-Botella y Palao, 2007). Con respecto a la dirección de lanzamiento, las zonas altas presentan mayor eficacia de lanzamiento que las zonas bajas (86\% frente 79\%), aunque también se observa una menor frecuencia de lanzamientos a estas zonas (22\% frente $78 \%)$.
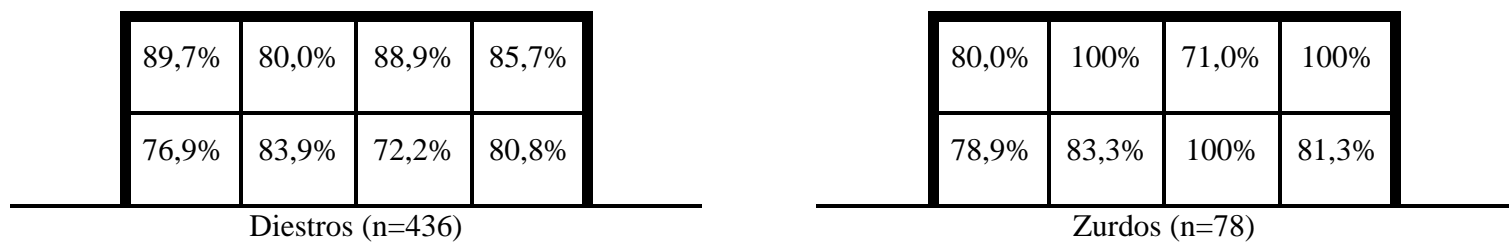

Figura 1. Porcentaje de eficacia en relación a la zona de lanzamiento para jugadores diestros y zurdos (López-Botella y Palao, 2007). 
Palau, J.M.; López-Montero, M.; López-Botella, M. (2010). Relación entre eficacia, lateralidad, y zona de lanzamiento del penalti en función del nivel de competición en fútbol. Revista Internacional de Ciencias del Deporte. 19(6), 154-165. http://www.cafyd.com/REVISTA/01905.pdf

No se ha encontrado en la revisión realizada si existen o no diferencias en la forma de ejecutar los penaltis en función del nivel de los lanzadores y del portero (nivel de competición). A este respecto existen estudios que indican que en diferentes deportes los jugadores más expertos o profesionales poseen mejores niveles de ejecución que los jugadores no expertos o amateurs (Castillo, Oña, Raya, y Martínez, 2005; Savelsbergh, Williams, Van der Kamp, y Ward, 2002; Williams, Davids, Burwitz, y Williams, 1994; Williams, y Davids, 1998). Esta misma tendencia se observa en el estudio de Jordet, Hartman, Visscher y Lemmink (2007) en lanzamiento de penaltis. Así, a mayor edad mayor eficacia de lanzamiento. No obstante debe tenerse en cuenta que la ejecución y el resultado del penalti depende del balance entre lanzador y portero. Es decir, aplicando la hipótesis de los trabajos anteriores, a menor nivel de competición tanto los lanzadores como los porteros serán de menor nivel lo que puede afectar o no sobre la forma de ejecución y el resultado del penalti. La ausencia de valores de referencia para los distintos niveles de competición hace que o bien los entrenadores no estén empleando información objetiva para el diseño de sus entrenamientos o bien se estén empleando valores del alto rendimiento. Por ello, el objetivo del presente trabajo fue conocer las diferencias existentes en función del nivel de competición en la lateralidad, la zona de lanzamiento y la eficacia del penalti en lanzadores especialistas profesionales de fútbol.

\section{Material y métodos}

\section{Muestra}

La muestra estudiada fue de 642 penaltis de competiciones profesionales españolas de $1^{\mathrm{a}}$ División ( $1^{\mathrm{a}}$ Div), $2^{\mathrm{a}}$ División A $\left(2^{\mathrm{a}} \mathrm{A}\right)$, y $2^{\mathrm{a}}$ División $\mathrm{B}\left(2^{\mathrm{a}} \mathrm{B}\right)$ de las temporadas 2004/2005, 2005/2006 y 2006/2007 (tabla 1). En total se analizaron 267 lanzadores (212 diestros y 55 zurdos).

Tabla 1. Número de lanzadores y de penaltis analizados en función de la competición (Temporadas 2004/05, 2005/06 y 2006/07).

\begin{tabular}{|l|c|c|c|c|c|c|}
\hline \multicolumn{1}{|c|}{ Competición } & \multicolumn{3}{c|}{ Número de lanzadores } & \multicolumn{2}{c|}{ Número de penaltis } \\
\hline & Diestros & $\%$ & Zurdos & $\%$ & Total & $\%$ \\
\hline $1^{\text {a }}$ División & 80 & 82.5 & 17 & 17.5 & 272 & $42.1 \%$ \\
\hline $2^{\text {a }}$ División A & 83 & 77.6 & 24 & 22.4 & 270 & $42.3 \%$ \\
\hline $2^{\text {a }}$ División B & 49 & 77.8 & 14 & 22.2 & 100 & $15.6 \%$ \\
\hline Totales & 212 & 79.4 & 55 & 20.6 & 642 & $100 \%$ \\
\hline
\end{tabular}

\section{Diseño}

Se realizó un diseño de investigación descriptivo correlacional intra e intergrupo. Se utilizó un sistema de categorías excluyentes como instrumento de observación (Anguera, 1989, Anguera, et al., 1993). Las variables objeto de estudio fueron:

a) Resultado del lanzamiento. Se registró el nivel de éxito del lanzamiento del penalti. Se distinguieron los siguientes resultados: a) Gol; b) Penalti parado o rechazado por el portero; c) Lanzamiento al poste o travesaño. La trayectoria del lanzamiento contacta directamente con el poste o travesaño de la portería; y d) Lanzamiento fuera. 
Palau, J.M.; López-Montero, M.; López-Botella, M. (2010). Relación entre eficacia, lateralidad, y zona de lanzamiento del penalti en función del nivel de competición en fútbol. Revista Internacional de Ciencias del Deporte. 19(6), 153-66. http://www.cafyd.com/REVISTA/01905.pdf

Observaciones: En el caso de que se produzca un rechace del portero o del poste, no se contabiliza acción posterior al lanzamiento aunque se consiga gol. Si el árbitro hace repetir el penalti, éste no se consideró en el estudio, únicamente se consideró el lanzamiento válido. En el caso de que no se produzca lanzamiento, no se contabiliza como tal (ej. pase a un compañero). Si el estado del terreno de juego hace que el balón no llegue a la portería, se registrará como balón parado en la zona correspondiente.

b) Lateralidad lanzador. Se registró el pie con el cual se golpea al balón por parte del lanzador del penalti. Se distinguieron las siguientes lateralidades: a) Diestro, y b) Zurdo.

c) Destino del lanzamiento. Se registró la zona de destino del lanzamiento. La zona de portería se dividió en ocho partes iguales y la zona de fuera de la portería se dividió en diez partes (figura 2). Se distinguieron las siguientes zonas tomando de referencia al portero situado en el centro de la portería: a) Zona 1 (1). Zona baja más alejada del lado derecho del portero; b) Zona 2 (2). Zona baja más próxima del lado derecho del portero; c) Zona 3 (3). Zona baja más próxima del lado izquierdo del portero; d) Zona 4 (4). Zona baja más alejada del lado izquierdo del portero; e) Zona 5 (5). Zona alta más alejada del lado izquierdo del portero; f) Zona 6 (6). Zona alta más próxima del lado izquierdo del portero; g) Zona 7 (7). Zona alta más próxima del lado derecho del portero; y h) Zona 8 (8). Zona alta más alejada del lado derecho del portero.

Observaciones: En caso de duda en la altura del lanzamiento, se observa la situación y posición del portero, la posición del cuerpo del portero en la "estirada", especialmente si su/s brazo/s están extendidos hacia arriba (zona alta) o si están semi-flexionados (zona baja). En caso de duda entre zona próxima y alejada al portero, se tuvieron las siguientes consideraciones: parte del cuerpo con la que se realiza la parada o rechace, la posición de los pies, y la colocación de los brazos en la actuación del portero.

En algunos casos, el dibujo de las mallas de la red ayuda a establecer las zonas de destino. Así como el retorno de la red, y dónde el balón quedó alojado en la red.

\begin{tabular}{ccc|c|c|c|} 
Zona 8 & Zona 8 & Zona 7 & Zona 6 & Zona 5 & Zona 5 \\
\cline { 2 - 5 } Zona 8 & Zona 8 & Zona 7 & Zona 6 & Zona 5 & Zona 5 \\
\cline { 2 - 5 } Zona 1 & Zona 1 & Zona 2 & Zona 3 & Zona 4 & Zona 4 \\
\hline
\end{tabular}

Figura 2. Distribución zonas de la portería.

d) Nivel de competición. Se registró el nivel de competición del lanzador. Se distinguieron los siguientes niveles: a) $1^{\text {a }}$ División; b) 2a División A; y c) 2a División B.

\section{Procedimiento}

La observación fue realizado por dos observadores que fueron entrenados de acuerdo a los criterios de Anguera (1989), Behar (1993) y Anguera, Blanco, Losada \& Hernández (2000). Después del entrenamiento y durante la observación de los penaltis, la fiabilidad interobservador e intra-observador fue calculada (Anguera, 1989). Todos los observadores tuvieron índices de confiabilidad superiores al 0.92.

\section{Estadística}

El análisis descriptivo e inferencial de los datos fue realizado con el software SPSS 13.0 (Test de Chi cuadrado y Test de razón de verosimilitudes). El nivel de significación se estableció en 0.05 .

\section{Resultados}


Palau, J.M.; López-Montero, M.; López-Botella, M. (2010). Relación entre eficacia, lateralidad, y zona de lanzamiento del penalti en función del nivel de competición en fútbol. Revista Internacional de Ciencias del Deporte. 19(6), 154-165. http://www.cafyd.com/REVISTA/01905.pdf

Con relación a la efectividad del lanzamiento, los lanzadores más efectivos fueron los de $2^{\mathrm{a}} \mathrm{A}$ $(77,4 \%)$ frente a los lanzadores de $1^{\mathrm{a}} \operatorname{Div}(73,9 \%)$ y $2^{\mathrm{a}} \mathrm{B}(69 \%)$. Las diferencias entre las competiciones fueron de un $4 \%$ entre $2^{\mathrm{a}}$ A y $1^{\mathrm{a}} \mathrm{Div}$, y de un $8 \%$ con $2^{\mathrm{a}} \mathrm{B}$. A nivel de lanzamientos fallados, en $1^{\text {a }}$ Div, el $70 \%$ de los lanzamientos fallados fueron parados por los porteros, el 13\% fueron poste o travesaño, y el 17\% fueron fuera. En $2^{\mathrm{a}} \mathrm{A}$, el $87 \%$ de los lanzamientos fallados fueron parados por los porteros, el 3\% fueron poste o travesaño, y el $10 \%$ fueron fuera. En $2^{\text {a }} \mathrm{B}$, el $81 \%$ los lanzamientos fallados fueron parados el portero, y el $10 \%$ fueron poste o travesaño. No se encontró significación estadística entre nivel de competición y eficacia del lanzamiento.

A nivel de zona de lanzamiento (derecha-izquierda del portero) y eficacia en función del nivel de competición (Figura 3), se observa que los diestros lanzaron en las tres competiciones más a la derecha del portero (59-62\% de los lanzamientos). Los zurdos de $1^{\text {a }}$ División lanzaron más a la derecha (59\%), los zurdos de $2^{\circ} \mathrm{A}$ y $2^{\circ} \mathrm{B}$ lanzaron más a la izquierda (68\%-60\%). Tanto los diestros como los zurdos fueron más efectivos lanzando a la derecha del portero. Esta tendencia no se observó en los lanzadores diestros de $2^{\mathrm{a}} \mathrm{A}$. No fue encontrada significación estadística entre lateralidad y zona de lanzamiento en ninguno de los niveles de competición analizados ni entre nivel de competición y eficacia del lanzamiento en función de la zona de lanzamiento (derecha-izquierda del portero).

$1^{\text {a }} \operatorname{Div}$
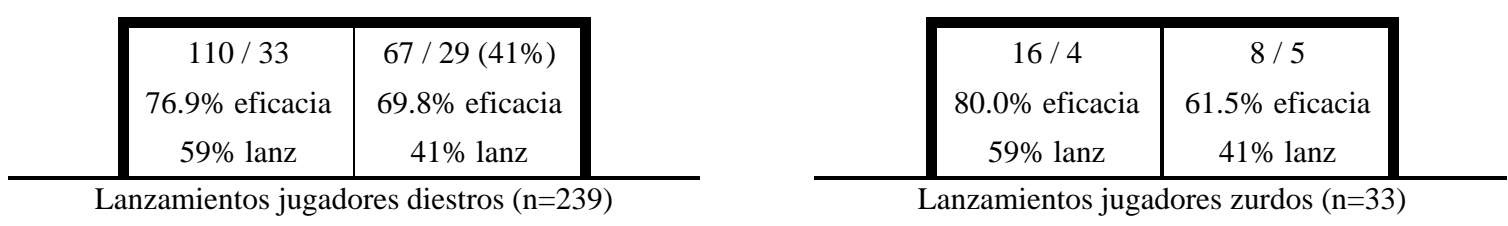

$2^{\mathrm{a}}$ Div A
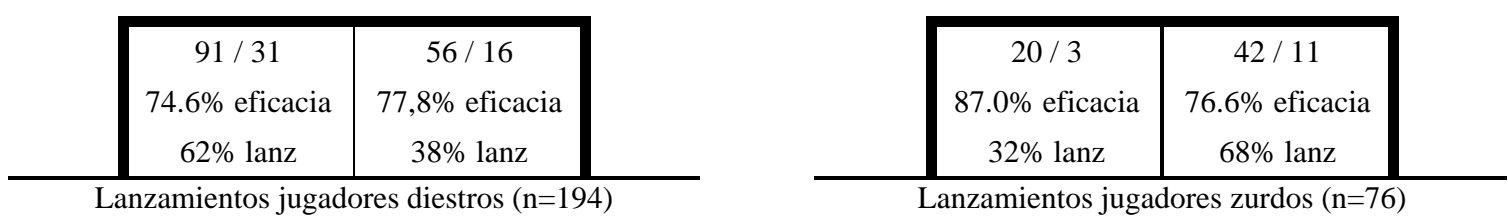

$2^{\text {a }}$ Div B
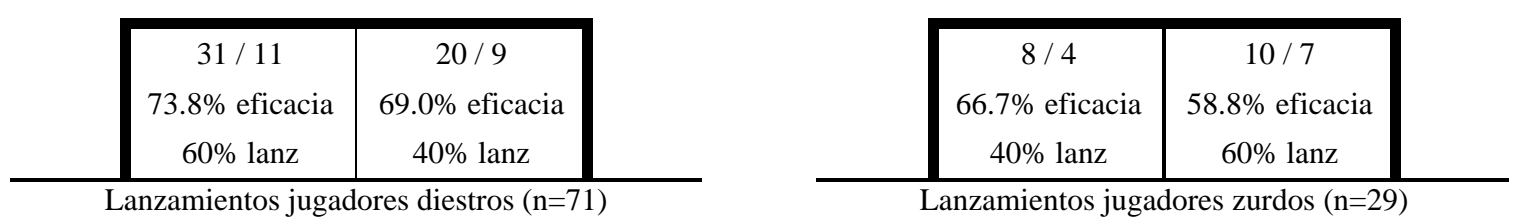

Figura 3. Goles, errores, y eficacia del lanzamiento de penalti en relación a la zona de lanzamiento (zona derecha y zona izquierda).

A nivel de zona de lanzamiento (arriba-abajo de la portería) y eficacia en función del nivel de competición (Figura 4), cuanto más bajo era el nivel de competición, los diestros lanzaron menos a las zonas altas de la portería (diferencia del 10\%). En los zurdos no se observó la misma tendencia. Así, en $2^{\mathrm{a}} \mathrm{A}$ se lanzó un $17 \%$, en $1^{\mathrm{a}}$ Div un $12 \%$, y en $2^{\mathrm{a}} \mathrm{B}$ un $7 \%$. A nivel de eficacia, tanto los diestros como los zurdos fueron más eficaces lanzando a las zonas altas que lanzando a las zonas bajas (12\% y 10\%, respectivamente). Esta tendencia no se observó en $1^{a}$ Div. En ninguno de los niveles de competición se paró ningún penalti en las zonas altas 
Palau, J.M.; López-Montero, M.; López-Botella, M. (2010). Relación entre eficacia, lateralidad, y zona de lanzamiento del penalti en función del nivel de competición en fútbol. Revista Internacional de Ciencias del Deporte. 19(6), 153-66. http://www.cafyd.com/REVISTA/01905.pdf

de la portería. Únicamente se encontraron diferencias estadísticamente significativas a nivel eficacia y de zona de lanzamiento en los lanzadores diestros de $2^{\circ}$ Div A $(\chi 2=16,441$; Significación $\mathrm{p}<0.001$ ), que presentan una tendencia a conseguir mayor eficacia en las zonas altas que en las zonas bajas.

$1^{\text {a }}$ Div
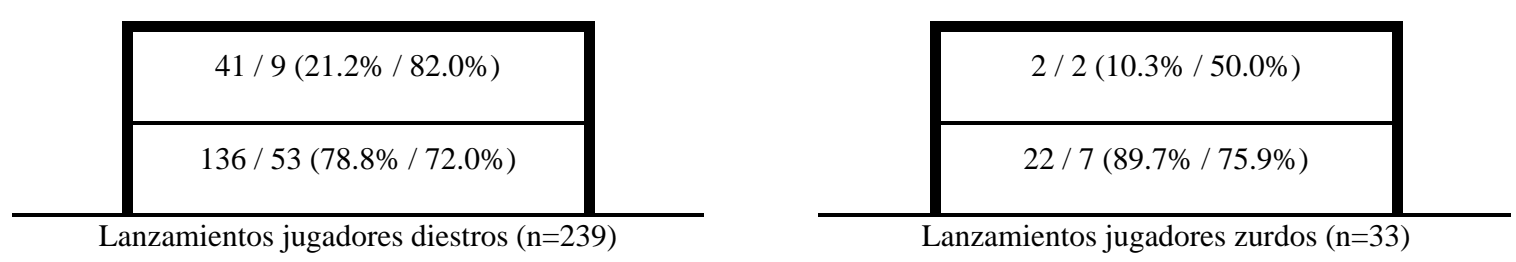

$2^{\text {a }}$ Div A
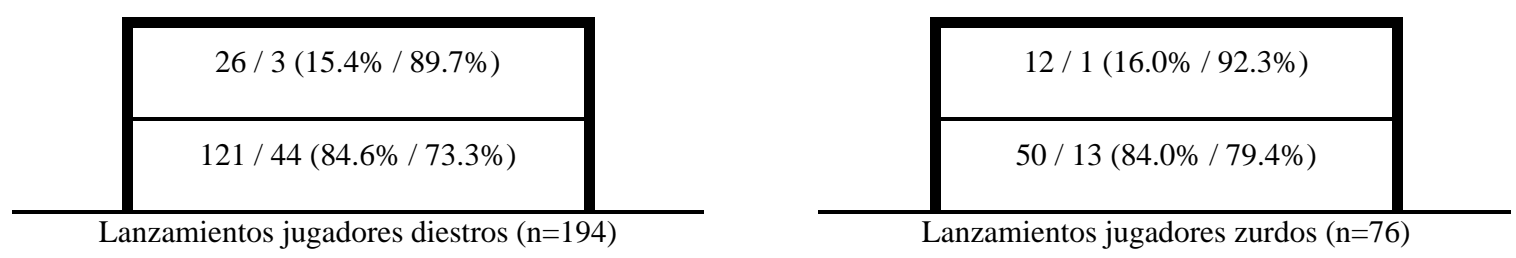

$2^{\mathrm{a}}$ Div B
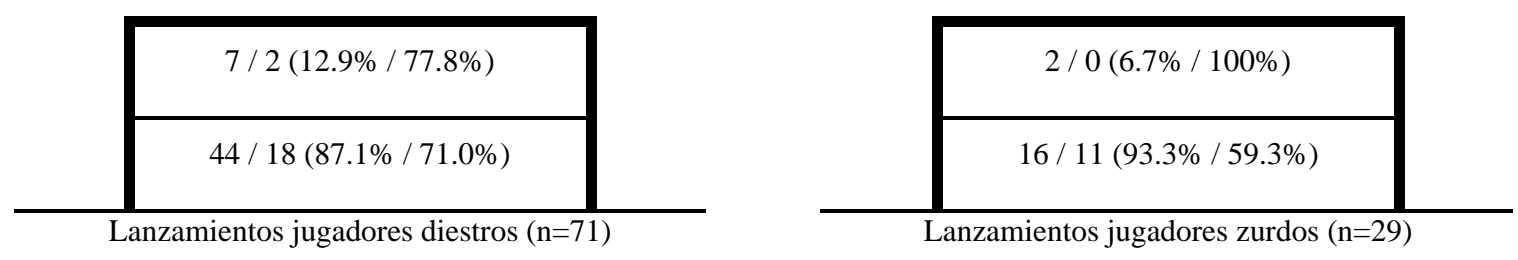

Figura 4. Goles, errores y eficacia del lanzamiento de penalti en relación a la zona de lanzamiento (zona alta y baja de la portería).

En relación a la zona de lanzamiento y eficacia en función del nivel de competición (Figura 5), las zonas bajas laterales de la portería fueron las que presentaron mayor porcentaje de ocurrencia. Dentro de estas zonas, para los diestros la zona que presentó mayor incidencia fue la zona 1 y para los zurdos fue la zona 4. A nivel de eficacia, por zonas, en los lanzadores diestros se observó que la efectividad en el lanzamiento a zona 1 fue la misma en las tres competiciones (72,0-73,5\%). En la zona 2, se observó una mayor eficacia en los lanzadores de $1^{\mathrm{a}}$ Div que los lanzadores de $2^{\mathrm{a}} \mathrm{A}$ y $2^{\mathrm{a}} \mathrm{B}$ (diferencias 13-14\%). En la zona 3, los lanzadores de $2^{\mathrm{a}} \mathrm{A}$ fueron un $8 \%$ más eficaces que los de $2^{\mathrm{a}} \mathrm{B}$. En la zona 4, fueron un $6 \%$ más efectivos en $2^{\mathrm{a}} \mathrm{A}$ que en $2^{\mathrm{a}} \mathrm{B}$. En la zona 5 , fueron un $50 \%$ más efectivos en $2^{\mathrm{a}} \mathrm{A}$ que en $2^{o}$ B. En la zona 6, fueron un $25 \%$ más efectivos en $2^{\mathrm{a}} \mathrm{A}$ y $2^{\mathrm{a}} \mathrm{B}$ que en $1^{\mathrm{a}}$ Div. En la zona 7 , fueron un $50 \%$ más efectivo en $1^{\mathrm{a}}$ Div que en $2^{\mathrm{a}} \mathrm{A}$. En la zona 8 , fueron un $15 \%$ más efectivos en $2^{\mathrm{a}} \mathrm{A}$ y $2^{\mathrm{a}} \mathrm{B}$ que en $1^{\mathrm{a}}$ Div. Se encontró una tendencia significativa a que se produjesen menos goles en los lanzamientos dirigidos a zona 4 realizados por los lanzadores de $1^{\text {a }}$ Div $\left(\chi^{2}=66,995\right.$; Significación $\left.\mathrm{p}<0.000\right)$, y una tendencia significativa a que se produjesen más goles en los lanzamientos dirigidos a zona 7 y 8 en $1^{\text {a }} \operatorname{Div}\left(\left(\chi^{2}=66,995\right.\right.$; Significación $\mathrm{p}<0.000)$ y en los lanzamientos dirigidos a zona 8 en $2^{\mathrm{a}} \operatorname{Div} \mathrm{A}\left(\chi^{2}=89,236\right.$; Significación $\mathrm{p}<0.000)$.

$1^{\mathrm{a}}$ Div 
Palau, J.M.; López-Montero, M.; López-Botella, M. (2010). Relación entre eficacia, lateralidad, y zona de lanzamiento del penalti en función del nivel de competición en fútbol. Revista Internacional de Ciencias del Deporte. 19(6), 154-165. http://www.cafyd.com/REVISTA/01905.pdf

\begin{tabular}{|c|c|c|c|}
\hline $84.6 \%$ & $100 \%$ & $75.0 \%$ & $63.6 \%$ \\
\hline $72.0 \%$ & $81.3 \%$ & $75.0 \%$ & $69.4 \%$ \\
\hline
\end{tabular}

\begin{tabular}{|c|c|c|c|}
\hline $100 \%$ & - & $0.0 \%$ & $100 \%$ \\
\hline $73.3 \%$ & $100 \%$ & $80.0 \%$ & $66.7 \%$ \\
\hline
\end{tabular}

$2^{\mathrm{a}}$ Div

\begin{tabular}{|l|l|l|l|}
\hline $100 \%$ & $50.0 \%$ & $100 \%$ & $100 \%$ \\
\hline $73.5 \%$ & $68.4 \%$ & $83.3 \%$ & $73.7 \%$ \\
\hline
\end{tabular}

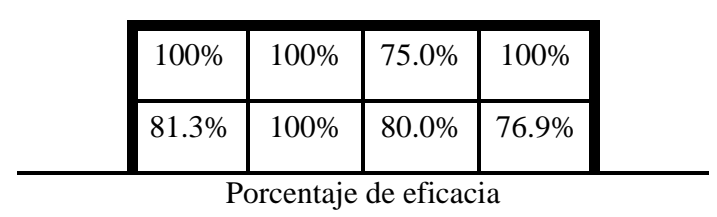

$2^{\mathrm{a}}$ Div B
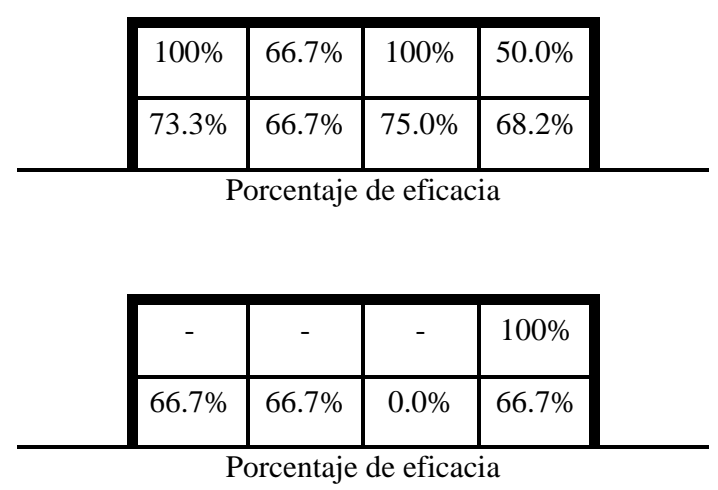
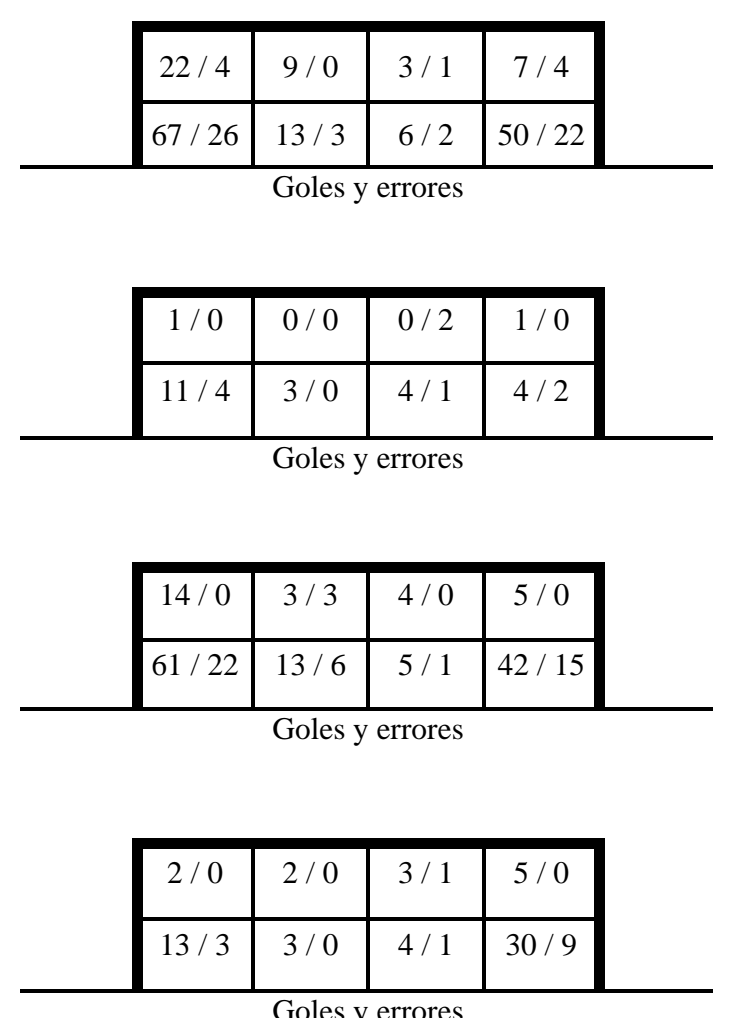

Goles y errores
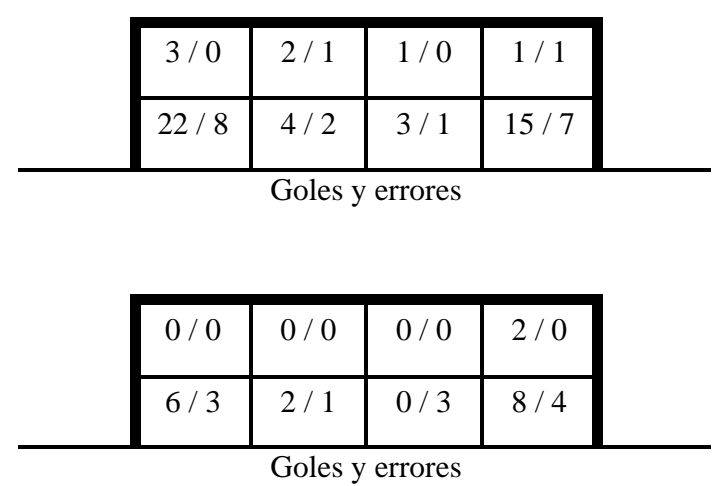

Figura 5. Goles, errores y eficacia del lanzamiento de penalti en relación a la zona de lanzamiento en lanzadores de $1^{\text {a }}$ División (239 diestros y 33 zurdos), 2 2a División A (194 diestros y 76 zurdos), y 2a División B (76 diestros y 71 zurdos).

Con respecto a los lanzadores zurdos, se observa que en la zona 1 fueron un 8\% más efectivos en $2^{\mathrm{a}} \mathrm{A}$ que en $1^{\mathrm{a}}$ Div y un $15 \%$ que en $2^{\mathrm{a}} \mathrm{B}$. En la zona 2 , fueron un $33 \%$ más efectivos en $1^{\mathrm{a}}$ Div y $2^{\mathrm{a}} \mathrm{A}$ que en $2^{\mathrm{a}} \mathrm{B}$. En la zona 3 (baja ocurrencia 3-5 lanz), en $2^{\mathrm{a}} \mathrm{B}$ lograron una efectividad del $0 \%$ y en $1^{a}$ Div y en $2^{\text {a }}$ A una efectividad del $80 \%$. En la zona 4 , fueron un $10 \%$ más efectivos en $2^{\mathrm{a}} \mathrm{A}$ que en $1^{\mathrm{a}}$ Div y $2^{\mathrm{a}} \mathrm{B}$. En la zona 5 , no hubo diferencias de efectividad en las tres competiciones (100\%). La zonas 6, 7, y 8 no presentaron ocurrencia $(0 \%)$. En todas las competiciones hubo zonas en las que se consiguieron el $100 \%$ de efectividad independientemente del pie de ejecución en el lanzamiento (aunque en algunas zonas el número de lanzamientos fue muy reducido). No se encontró significación estadística entre nivel de competición y eficacia del lanzamiento en jugadores zurdos. 
Palau, J.M.; López-Montero, M.; López-Botella, M. (2010). Relación entre eficacia, lateralidad, y zona de lanzamiento del penalti en función del nivel de competición en fútbol. Revista Internacional de Ciencias del Deporte. 19(6), 153-66. http://www.cafyd.com/REVISTA/01905.pdf

\section{Discusión}

Los resultados encontrados muestran que los lanzadores más eficaces no fueron los que juegan en mayor nivel de competición, aunque las diferencias encontradas son reducidas, del 3-8\%. Estos datos son contrarios a los encontrados por lanzadores especialistas y tandas en las Copas del Mundo 1976-2004 (Jordet, Hartman, Lemmink y Visscher, 2007). Estas diferencias pueden deberse a que en el presente trabajo sólo se estudiaron lanzadores especialistas. Otro aspecto al que pueden deberse estas diferencias, y que no se ha considerado en el presente estudio es que el resultado del lanzamiento del penalti depende del balance portero-lanzador. No es posible ratificar que el balance entre el nivel de los lanzadores y de los porteros de las tres competiciones sea el mismo o se varíe.

Los niveles de eficacia encontrados fueron inferiores a los hallados en estudios previos en especialistas (López-Botella y Palao, 2007) y similares a los encontrados en lanzadores de tandas (Hughes y Wells, 2001; Miller, 1996; Vázquez, 1998; Castillo et al., 2000; Morya, et al., 2003). A partir de estos datos se extrae que el $96 \%$ en $1^{\text {a }}$ Div, el $97 \%$ en $2^{\text {a }}$ B y el $98 \%$ en $2^{a}$ A son dirigidos a la superficie que ocupa la portería incluidos postes y travesaños. Estos datos son similares a los encontrados en ligas profesionales de $1^{\text {a }}$ Div de competiciones europeas y sudamericanas (López-Botella y Palao, 2007).

Con respecto a la lateralidad, los resultados muestran valores similares entre jugadores diestros y zurdos en $1^{\circ}$ Div. En $2^{\circ} \mathrm{A}$, los zurdos fueron los lanzadores que presentaron mayor eficacia (82\%). En $2^{\circ} \mathrm{B}$, los diestros fueron los que obtienen más eficacia (71\%). Estos resultados no permiten extraer conclusiones o tendencias claras entre el nivel de los lanzadores y la lateralidad en función del nivel de competición. Son necesarias más investigaciones que incluyan aspectos no controlados en este estudio.

Los resultados encontrados mostraron una tendencia a lanzar a un lado u otro de la portería en función de la lateralidad. Así, los diestros tendieron a lanzar al lado derecho del portero, y los zurdos tendieron a lanzar al lado izquierdo del portero. Esta tendencia no se cumplió en los jugadores zurdos de $1^{\circ}$ Div. Esto puede mostrar que hay jugadores que tienen la capacidad de variar su ejecución. Los resultados encontrados confirman las tendencias de zona de lanzamiento en diestros (Raya y Navarro, 1990; López-Botella y Palao, 2007) y en zurdos (López-Botella y Palao, 2007). A nivel de eficacia, se observa un mejor rendimiento de los lanzadores cuando dirigen los lanzamientos al lado derecho del portero tanto en diestros como en zurdos (diferencias 6-12\%). Esta tendencia no se observó en $2^{\circ}$ A. En este nivel de competición, la eficacia de lanzamiento en ambas direcciones fue similar. Un estudio más específico es necesario para conocer las causas de estas diferencias.

A nivel de altura de lanzamiento, se observó que a mayor nivel de competición se produce un mayor porcentaje de lanzamientos a la zona alta de la portería. Se encontró una menor tendencia de los zurdos a lanzar a las zonas altas. Los zurdos de $2^{\circ} \mathrm{A}$ presentaron valores similares que los diestros. No obstante, la realización de lanzamientos a esta zona, aunque presentó una mayor eficacia tiene un bajo porcentaje de utilización (10-20\%). Así, ocho de cada diez lanzamientos dirigidos a la zona alta son gol. En esta línea, cabe destacar que ninguno de los lanzamientos dirigidos a esta zona fue parado por el portero. Los resultados encontrados son ligeramente superiores a los encontrados en estudios previos (López-Botella y Palao, 2007) en diestros ( $8 \%$ frente a $12 \%$ ) mientras que en los zurdos son inferiores (2\% frente $10 \%)$. 
Palau, J.M.; López-Montero, M.; López-Botella, M. (2010). Relación entre eficacia, lateralidad, y zona de lanzamiento del penalti en función del nivel de competición en fútbol. Revista Internacional de Ciencias del Deporte. 19(6), 154-165. http://www.cafyd.com/REVISTA/01905.pdf

A nivel de zona de lanzamiento, los resultados encontrados van en la misma línea que los antes indicados, se observó una tendencia de los jugadores a lanzar a las zonas bajas de la portería. En función de la lateralidad, en diestros la tendencia fue lanzar al lado derecho del portero (zona 1) y en zurdos la tendencia fue lanzar al lado izquierdo del portero (zona 4). De forma específica por zonas no se observaron tendencias claras por zonas y/o lateralidad, salvo las indicadas con anterioridad. Estos resultados pueden indicar que es necesario realizar el estudio por zonas específicas y tener en cuenta las características individuales del lanzador y su forma de lanzar.

A partir de los resultados obtenidos se pueden extraer las siguientes conclusiones:

- Los lanzadores de penaltis analizados obtienen gol en siete de cada 10 lanzamientos.

- Los diestros tienden a lanzar a la derecha del portero, y los zurdos a la izquierda del portero. Tanto diestros como zurdos fueron más efectivos lanzando a la derecha del portero.

- Los lanzamientos dirigidos a las zonas altas presentaron un $10 \%$ mayor de eficacia que los lanzamientos dirigidos a las zonas bajas. Ninguno de los lanzamientos dirigidos a las zonas altas de la portería fue parado por el portero. A menor nivel de la competición, menos lanzamientos a las zonas altas.

Se debe tener en cuenta que al obtener estas conclusiones no se valoró cuál fue la forma de lanzamiento del jugador, ni considerándose si lanzaba teniendo en cuenta las acciones del portero.

\section{Aplicaciones al entrenamiento}

Los valores obtenidos pueden servir de base para el establecimiento de objetivos de eficacia del entrenamiento de especialistas en el lanzamiento del penalti. A nivel de eficacia, tanto diestros como zurdos deben conseguir al menos siete goles de cada diez lanzamientos que realicen. En relación a las zonas (figura 6), a partir de los datos obtenidos, a los diestros se les recomienda entrenar los lanzamientos a la zona 8 y a los zurdos a la zona 5. Las zonas no recomendadas son la zona 5 en $1^{\mathrm{a}} \mathrm{Div}$, la zona 7 en $2^{\mathrm{a}} \mathrm{A}$, y la zona 5 en $2^{\mathrm{a}} \mathrm{B}$ para los diestros. Para los zurdos la zona 6 en $1^{\mathrm{a}}$ Div y $2^{\mathrm{a}} \mathrm{A}$ y la zona 3 en $2^{\mathrm{a}} \mathrm{B}$.

En el establecimiento de los objetivos de entrenamiento se deben considerar también las características y tendencias de los lanzadores que se entrenan. A modo de ejemplo, vamos a coger a dos lanzadores diestros de $1^{\text {a }}$ Div y que son especialistas en sus equipos (Figura 7). En estos lanzadores tomados de referencia se observa que ambos tienen una eficacia diferente (Jugador A, un 91\% y Jugador B un 50\%) y tendencias a lanzar a diferentes zonas de la portería. En el caso del Jugador A se observa una tendencia definida mientras que esto no ocurre en el caso del Jugador B. La efectividad del Jugador A está por encima de la media de $1^{\mathrm{a}}$ Div mientras que el Jugador B se encuentra por debajo. 
Palau, J.M.; López-Montero, M.; López-Botella, M. (2010). Relación entre eficacia, lateralidad, y zona de lanzamiento del penalti en función del nivel de competición en fútbol. Revista Internacional de Ciencias del Deporte. 19(6), 153-66. http://www.cafyd.com/REVISTA/01905.pdf

$1^{\mathrm{a}} \mathrm{Div}$
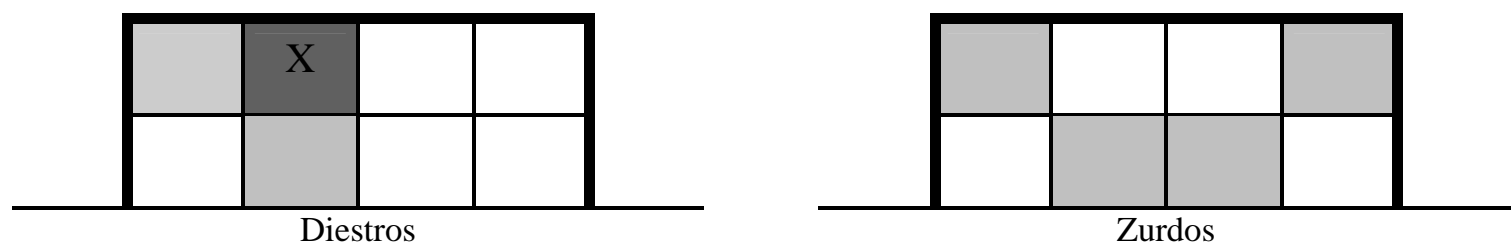

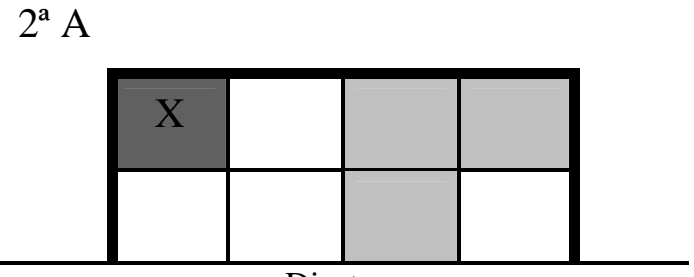

Diestros

$2^{\mathrm{a}} \mathrm{B}$

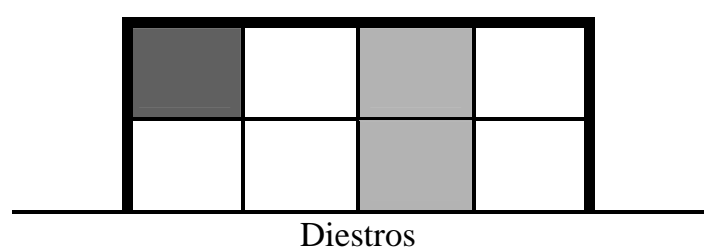

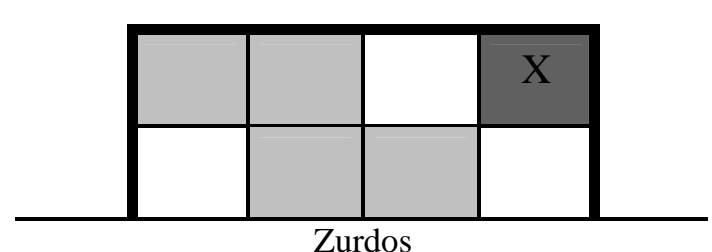

Zurdos

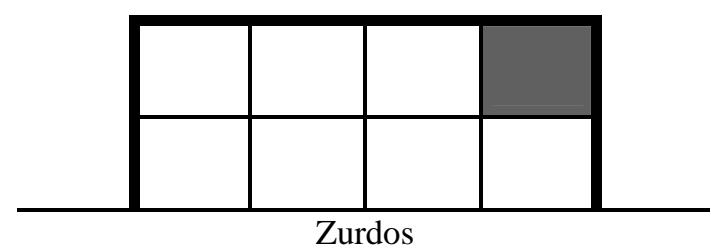

Figura 6. Recomendaciones de zonas con mayor eficacia en $1^{\mathrm{a}}$ División, $2^{\mathrm{a}}$ División A, y 2 2a División B (España).

En el primer caso, el Jugador A, que es diestro, muestra una clara y eficaz tendencia a la zona derecha del portero (zona 1 y 8 ). Teniendo en cuenta que su efectividad en la zona 8 es del $100 \%$ su entrenamiento tiene que tener como objetivo la eficacia que ya tiene en esta zona. La zona 1 con $80 \%$ de eficacia también debe ser trabajada aunque es más accesible para los porteros. Este jugador debe trabajar con unos niveles de eficacia superiores al $80 \%$, no siendo adecuado trabajar con él, tomando de referencia los valores medios del resto de los lanzadores.
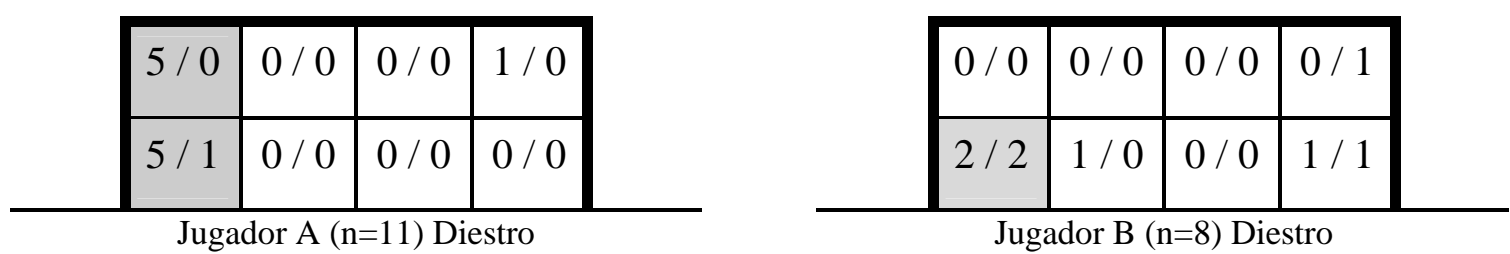

Figura 7. Ejemplo de goles y errores por zona de portería de dos jugadores de 1ª División Española.

En el segundo caso, Jugador B, jugador diestro, tiene una ligera preferencia a lanzar a la derecha del portero. En los penaltis estudiados, su nivel de eficacia está por debajo de la media en $1^{a}$ Div en cuanto eficacia. El primer paso es realizar un trabajo de mejora en base. Para ello se debe conocer cuál es/son la/s zona/s adecuada/s para este jugador. Por ello, se deben realizar lanzamientos a cada una de las zonas sin portero (ej. 10 a cada una de las 
Palau, J.M.; López-Montero, M.; López-Botella, M. (2010). Relación entre eficacia, lateralidad, y zona de lanzamiento del penalti en función del nivel de competición en fútbol. Revista Internacional de Ciencias del Deporte. 19(6), 154-165. http://www.cafyd.com/REVISTA/01905.pdf

zonas). A partir de esta prueba se deben decidir dos zonas en las cuales practicar sin portero y con portero.

Nota: Este planteamiento es válido si el jugador lanza sin mirar al portero en el golpeo.

Tabla 2. Ejemplo de propuesta de entrenamiento individualizado en relación a la zona de lanzamiento.

\begin{tabular}{|c|l|}
\hline Jugador & Propuesta de entrenamiento individualizado \\
\hline \multirow{3}{*}{ Jugador A } & $\begin{array}{l}\text { Trabajar con una eficacia superior al 80\%. } \\
\text { Entrenamiento sin portero tandas de precisión a la zona } 8 \text { y 1. En algún caso a la zona } \\
\text { Entrenamiento con portero lanzamientos a la zona } 8 \text { y 1. En algún caso a las zonas } \\
\text { opuesta (zona } 5 \text { o zona 1). } \\
* \text { En cada lanzamiento cambiar de portero. }\end{array}$ \\
& $\begin{array}{l}\text { Planteamiento para un lanzador que no mira la portería al golpear. } \\
\text { Buscar mantener una eficacia superior al 70\%. } \\
\text { Establecer las zonas más adecuadas al jugador. Entrenamiento sin portero. } \\
\text { Entrenamiento con portero. } \\
* \text { En cada lanzamiento cambiar de portero. }\end{array}$ \\
\hline
\end{tabular}

A modo de referencia para el establecimiento de los objetivos en el entrenamiento del penalti se deben tener en cuenta los siguientes aspectos:

Nivel de efectividad en función del nivel de competición y de la lateralidad de los jugadores.

Z Zonas de la portería que son más eficaces en función de la lateralidad de los jugadores.

> Conocer el nivel de precisión sin portero de los jugadores para saber quién o quiénes son los lanzadores adecuados del equipo.

$>$ Trabajar lanzamientos con portero (no tandas) a las zonas elegidas como más efectivas con zonas y eficacia establecida.

$>$ Crear situaciones de lanzamiento del penalti similares a las que se dan en competición (ej. cuando se consigue gol, para que este suba realmente al marcador se debe lanzar un penalti y obtener gol). 


\section{Referencias}

Anguera, M. T., Behar J., Blanco, A., Carreras, M. V., Losada, J. L., Quera, V. y Riba, C. (1993). Metodología observacional en la investigación psicológica (Volumen I). Barcelona: Promociones y publicaciones Universitarias.

Anguera, M. T., Blanco, A., Losada, J. L. y Hernández, A. (2000). La metodología observacional en el deporte: Conceptos básicos [Versión electrónica]. EF Deportes, 24. <http://www.efdeportes.com/efd24b/obs.htm>.

Anguera, M.T. (1989). Metodología de observación en las ciencias humanas. Cátedra. Madrid.

Behar, J. (1993). Sesgos del observador (Capitulo 1). En M.T. Anguera (Ed.) Metodología observacional en la investigación psicológica (Volumen II). (pp. 27-76). Barcelona: Promociones y publicaciones Universitarias.

Castillo J. M., Oña, A., Raya, A. y Martínez, M. A. (2005). Visual training of expert and intermediate penalty kickers in soccer. J. Hum. Movement Stud., 49, 255-275

Castillo, J. M., Oña, A., Raya, A. y Martínez, M. A. (2002). Aplicación de un sistema automatizado para lanzadores de penalty en fútbol. Motricidad, 8, 73-94.

Hughes, M. y Wells, J. (2001). Analysis of penalties taken in shoot-outs. International J ournal of Performance Analysis in Sport, 2(1), 55-72.

Jordet, G., Hartman, E., Visscher, C. y Lemmink, K. (2007). Kicks from the penalty mark in soccer: The roles of stress, skill, and fatigue for kick outcomes. Journal of Sports Sciences, 25(2): 121-129.

López-Botella, M. y Palao, J. M. (2007). Relationship between laterality of foot strike and shot zone on penalty efficacy in specialist penalty takers. International Journal of Performance Analysis in Sport, 7(3), 26-36.

Miller, R. (1996). Shooter vs keeper: a tense battle. Games are won or lost by penalty kicks and PK tie breakers. Soccer Journal, 7, 59-62.

Morya, E., Bigatao, H., Lees, A. y Ranvaud, R. (2003). Evolving penalty kick strategies: World Cup and club matches 2000-2002. En Science and Football 5th World Congress (editado por Technical University of Lisbon), pp. 47-48. Madrid: Gymnos.

Raya, A. y Navarro, M. (1990). Análisis de la acción técnico-táctica del portero ante el penalti. Análisis de las zonas del campo y de la superficie de contacto con la que se produjeron los goles (World Cup México 1986). Cuaderno del entrenador. 45, pp. 2535.

Vázquez, S. (1998). ¿El penalti futbolístico es castigo o condena?. Cuaderno del entrenador. 78, 44-57.

Williams, A. M. y Davids, K. (1998). Visual search strategy, selective attention, and expertise in soccer. Res. Q. Exer. Sport, 69, 11-128.

Williams, A. M., Davids, K., Burwitz, L. y Williams, J. G. (1994). Visual search strategies in experienced and inexperienced soccer players. Res. Q. Exer. Sport, 65, 127-135.

Williams, A. M., van der Kamp, J. y Ward, P. (2002) Visual search, anticipation and expertise in soccer goalkeepers'. J ournal of Sports Sciences. 20, 279-287. 